\title{
Perinatal factors associated with early deaths of preterm infants born in Brazilian Network on Neonatal Research centers
}

\author{
Fatores perinatais associados ao óbito precoce em prematuros nascidos nos \\ centros da Rede Brasileira de Pesquisas Neonatais \\ Maria Fernanda Branco de Almeida ${ }^{1}$, Ruth Guinsburg ${ }^{2}$, Francisco Eulógio Martinez ${ }^{3}$, \\ Renato S. Procianoy4, Cléa Rodrigues Leone ${ }^{5}$, Sérgio Tadeu Martins Marba ${ }^{6}$, \\ Lígia Maria Sousa Suppo Rugolo7, Jorge Hecker Luz ${ }^{8}$, José Maria de Andrade Lopes ${ }^{9}$
}

\section{Resumo}

Objetivo: Avaliar os fatores perinatais associados ao óbito neonatal precoce em prematuros com peso ao nascer entre 400 e $1.500 \mathrm{~g}$.

Métodos: Coorte prospectiva e multicêntrica dos nascidos vivos com idade gestacional de 23 a 33 semanas e peso de $400-1.500 \mathrm{~g}$, sem malformações em oito maternidades públicas terciárias universitárias entre junho de 2004 e maio de 2005. As características maternas e neonatais e a morbidade nas primeiras 72 horas de vida foram comparadas entre os prematuros que morreram ou sobreviveram até o sexto dia de vida. As variáveis perinatais associadas ao óbito neonatal precoce foram determinadas por regressão logística.

Resultados: No período, 579 recém-nascidos preencheram os critérios de inclusão. O óbito precoce ocorreu em 92 (16\%) neonatos, variando entre as unidades de 5 a $31 \%$, e tal diferença persistiu controlando-se por um escore de gravidade clínica (SNAPPE-II). A análise multivariada para o desfecho óbito neonatal intra-hospitalar precoce mostrou associação com: idade gestacional de 23-27 semanas (odds ratio - OR $=5,0$; IC95\% 2,7-9,4), ausência de hipertensão materna $\left(\mathrm{OR}=1,9\right.$; IC95\% 1,0-3,7), Apgar 0-6 no $5^{\circ}$ minuto $(\mathrm{OR}=$ 2,$8 ;$ IC95\% 1,4-5,4), presença de síndrome do desconforto respiratório $(\mathrm{OR}=3,1$; IC95\% 1,4-6,6) e centro em que o paciente nasceu.

Conclusão: Importantes fatores associados ao óbito neonatal precoce em prematuros de muito baixo peso são passíveis de intervenção, como a melhora da vitalidade fetal ao nascer e a diminuição da incidência e gravidade da síndrome do desconforto respiratório. As diferenças de mortalidade encontradas entre os centros apontam para a necessidade de identificar as melhores práticas e adotá-las de maneira uniforme em nosso meio.

J Pediatr (Rio J). 2008;84(4):300-307: Mortalidade neonatal; recém-nascido; prematuro; recém-nascido de muito baixo peso.

\section{Abstract}

Objective: To evaluate perinatal factors associated with early neonatal death in preterm infants with birth weights (BW) of 400-1,500 g.

Methods: A multicenter prospective cohort study of all infants with BW of 400-1,500 g and 23-33 weeks of gestational age (GA), without malformations, who were born alive at eight public university tertiary hospitals in Brazil between June of 2004 and May of 2005. Infants who died within their first 6 days of life were compared with those who did not regarding maternal and neonatal characteristics and morbidity during the first 72 hours of life. Variables associated with the early deaths were identified by stepwise logistic regression.

Results: A total of 579 live births met the inclusion criteria. Early deaths occurred in $92(16 \%)$ cases, varying between centers from 5 to $31 \%$, and these differences persisted after controlling for newborn illness severity and mortality risk score (SNAPPE-II). According to the multivariate analysis, the following factors were associated with early intrahospital neonatal deaths: gestational age of 23-27 weeks (odds ratio $-\mathrm{OR}=5.0 ; 95 \% \mathrm{CI} 2.7-9.4)$, absence of maternal hypertension $(\mathrm{OR}=1.9 ; 95 \% \mathrm{CI} 1.0-3.7), 5$ th minute Apgar $0-6(\mathrm{OR}=2.8 ; 95 \% \mathrm{CI}$ $1.4-5.4)$, presence of respiratory distress syndrome $(\mathrm{OR}=3.1 ; 95 \% \mathrm{CI}$ 1.4-6.6), and network center of birth.

Conclusion: Important perinatal factors that are associated with early neonatal deaths in very low birth weight preterm infants can be modified by interventions such as improving fetal vitality at birth and reducing the incidence and severity of respiratory distress syndrome. The heterogeneity of early neonatal rates across the different centers studied indicates that best clinical practices should be identified and disseminated throughout the country.

J Pediatr (Rio J). 2008;84(4):300-307: Neonatal mortality, infant, newborn, infant, preterm, infant, very low birth weight.

1. Professora associada, Disciplina de Pediatra Neonatal, Universidade Federal de São Paulo - Escola Paulista de Medicina (UNIFESP-EPM), São Paulo, SP.

2. Professora titular, Disciplina de Pediatra Neonatal, UNIFESP-EPM, São Paulo, SP.

3. Professor titular, Departamento de Pediatria, Faculdade de Medicina de Ribeirão Preto, Universidade de São Paulo (USP), Ribeirão Preto, SP.

4. Professor titular, Departamento de Pediatria, Faculdade de Medicina, Universidade de Federal do Rio Grande do Sul (UFRGS), Porto Alegre, RS.

5. Professora associada, livre-docente, Departamento de Pediatria, Faculdade de Medicina, USP, São Paulo, SP.

6. Professor livre-docente, Departamento de Pediatria, Faculdade de Ciências Médicas, Universidade Estadual de Campinas (UNICAMP), Campinas, SP.

7. Professora adjunta, livre-docente, Departamento de Pediatria, Faculdade de Medicina de Botucatu, Universidade Estadual Paulista (UNESP), Botucatu, SP.

8. Mestre, Pontifícia Universidade Católica do Rio Grande do Sul (PUCRS), Porto Alegre, RS.

9. Doutor. Chefe, Clínica de Neonatologia, Instituto Fernandes Figueira, Fundação Osvaldo Cruz (FIOCRUZ), Rio de Janeiro, RJ.

Este estudo foi realizado pela Rede Brasileira de Pesquisas Neonatais.

Apoio financeiro: Ministério da Saúde, Brasil.

Não foram declarados conflitos de interesse associados à publicação deste artigo.

Como citar este artigo: de Almeida MF, Guinsburg R, Martinez FE, Procianoy RS, Leone CR, Marba ST, et al. Perinatal factors associated with early deaths of preterm infants born in Brazilian Network on Neonatal Research centers. J Pediatr (Rio J). 2008;84(4):300-307.

Artigo submetido em 19.12.07, aceito em 27.03.08.

doi:10.2223/JPED. 1787 


\section{Introdução}

A sobrevivência de recém-nascidos prematuros e de muito baixo peso reflete a qualidade do atendimento antenatal, do cuidado ao trabalho de parto e parto e a estrutura de atendimento neonatal das diversas regiões e países do mundo. Assim, é de se esperar que países mais ricos apresentem taxas de mortalidade neonatal precoce e tardia inferiores às de países nos quais a atenção à saúde é mais precária. Por outro lado, a freqüência de prematuridade é maior nos países mais pobres devido justamente às condições mais precárias de saúde da gestante ${ }^{1}$. O parto prematuro é o determinante mais importante da mortalidade infantil nos países desenvolvidos $^{2}$. Para os partos hospitalares dos países em desenvolvimento, a prematuridade também é o principal determinante da morbidade e mortalidade neonatal ${ }^{3}$.

Nos EUA, em 2004, os nascimentos abaixo de 28 semanas corresponderam a $0,8 \%$ de todos os nascidos vivos e a $46,3 \%$ das mortes infantis ${ }^{2}$. É preocupante o aumento notado na freqüência de prematuridade no mundo, com a possível exceção da França e Finlândia ${ }^{4}$. No Brasil, em 2004, houve 3.026 .548 nascimentos, dos quais $34.012(1,1 \%)$ com peso $<1.500 \mathrm{~g}^{5}$. Nesse mesmo ano, morreram 54.183 crianças antes de 1 ano de vida, sendo 15.560 (29\%) com peso ao nascer $<1.500 \mathrm{~g}$ e, destes óbitos, $11.426(73 \%)$ faleceram antes de completar 7 dias de vida ${ }^{6}$.

Para avaliar com mais profundidade a mortalidade neonatal precoce de recém-nascidos prematuros em nosso meio, deve-se levar em conta o contexto no qual os dados foram obtidos, ou seja, a qualidade do cuidado antenatal, do atendimento ao trabalho de parto e parto, a infra-estrutura em termos de recursos físicos, materiais e humanos e a prevalência do emprego de intervenções baseadas em evidências, além da participação familiar no cuidado intensivo neonatal ${ }^{7}$. Neste sentido, as redes epidemiológicas, que buscam analisar os dados de morbidade e mortalidade de diversas unidades neonatais, são capazes de fornecer dois tipos de informação. Por um lado, estas redes podem retratar em tempo real a morbidade e mortalidade neonatal em centros com características específicas e comparar estes dados aos nacionais e internacionais. Por outro lado, ao permitir a comparação entre os diversos centros, as informações obtidas possibilitam estudar as melhores estratégias para diminuir a mortalidade e a morbidade neonatal, baseadas em processos de melhoria da qualidade da assistência perinatal ${ }^{8,9}$. É nesse contexto que se coloca o presente trabalho, cujo objetivo foi avaliar os fatores perinatais associados ao óbito até o sexto dia de vida em prematuros de muito baixo peso assistidos em hospitais universitários públicos nos estados de São Paulo, Rio de Janeiro e Rio Grande do Sul, com ênfase nos diferentes perfis dos pacientes atendidos nos diversos hospitais.

\section{Métodos}

Trata-se de uma coorte prospectiva de todos os nascidos vivos com idade gestacional de 23 0/7 a 33 6/7 semanas e peso de 400-1.500 g nascidos em oito maternidades públicas terciárias e universitárias, localizadas em cinco cidades de três estados brasileiros (RJ, RS e SP), entre junho de 2004 e maio de 2005. Foram excluídos do estudo os pacientes portadores de malformações congênitas maiores e aqueles transferidos de outras instituições. Todas as maternidades são referência para gestação de alto risco, atendem quase que exclusivamente pacientes do Sistema Único de Saúde e oferecem programas de residência médica em pediatria e em neonatologia. O projeto foi aprovado pelo Comitê de Ética em Pesquisa de cada uma das oito instituições. Para manter a confidencialidade dos dados, estas instituições são denominadas de unidades $\mathrm{A}$ até $\mathrm{H}$.

As oito unidades possuem sala de parto com recursos para a reanimação neonatal e, no total, contam com 282 leitos de terapia intensiva e cuidados intermediários, variando de 20 a 48 leitos por unidade. Além da estrutura física para o cuidado do recém-nascido de alto risco, todas contam com recursos materiais e humanos para a assistência intensiva, de acordo com as normas do Ministério da Saúde do Brasil e da Sociedade Brasileira de Pediatria ${ }^{10}$.

Em cada unidade, um a dois neonatologistas coletaram de forma prospectiva, por meio de um questionário específico para a realização do trabalho aplicado às puérperas e aos recém-nascidos, informações relativas aos seguintes aspectos:

Características maternas: idade e escolaridade em anos; assistência pré-natal, classificada em presente ou ausente (não se levou em conta o número de consultas por se tratar de uma população de grávidas com idade gestacional inferior a 34 semanas); gestação única ou múltipla; síndrome hipertensiva de qualquer etiologia e diabetes, consideradas presentes quando diagnosticadas pelo obstetra; infecção periparto, definida diante de processo infeccioso de qualquer etiologia na semana anterior ao parto; corticóide pré-natal (presente se pelo menos uma dose de dexametasona ou betametasona foi aplicada antes do parto) e tipo de parto.

Características do paciente ao nascimento: idade gestacional determinada pela melhor estimativa obstétrica ou pelo exame físico do recém-nascido ${ }^{11}$; peso mensurado em balança com precisão de $5 \mathrm{~g}$; adequação peso/idade gestacional, definindo-se os pequenos para idade gestacional como aqueles abaixo do percentil 10 da curva de crescimento intrauterino $^{12}$; sexo; boletim de Apgar com 1 e 5 minutos; procedimentos de reanimação segundo as normas da Academia Americana de Pediatria, em $2000^{13}$ (ventilação com pressão positiva = ventilação com balão e máscara ou cânula traqueal e reanimação avançada = ventilação acompanhada de massagem cardíaca e/ou medicações). Coletou-se também informação referente à instilação de surfactante na sala de parto.

Evolução clínica nas primeiras $\mathbf{7 2}$ horas de vida: Score for Neonatal Acute Physiology, Perinatal Extension, Version II (SNAPPE-II), definindo-se como prognóstico pior aqueles com escore $>39^{14}$; síndrome do desconforto respiratório diagnosticada segundo critérios clínicos e radiológicos; persistência do canal arterial definida por alterações clínicas e/ou ecocardiográficas; sepse caracterizada por critérios clínicos 
Tabela 1 - Características demográficas maternas dos prematuros nascidos nas oito unidades avaliadas

\begin{tabular}{|c|c|c|c|c|c|c|c|c|c|}
\hline & $\begin{array}{c}\text { Unidade } \\
\text { A } \\
(n=41)\end{array}$ & $\begin{array}{c}\text { Unidade } \\
\text { B } \\
(n=68)\end{array}$ & $\begin{array}{c}\text { Unidade } \\
\text { C } \\
(n=66)\end{array}$ & $\begin{array}{c}\text { Unidade } \\
\text { D } \\
(n=85)\end{array}$ & $\begin{array}{c}\text { Unidade } \\
\text { E } \\
(n=86)\end{array}$ & $\begin{array}{c}\text { Unidade } \\
\text { F } \\
(n=41)\end{array}$ & $\begin{array}{c}\text { Unidade } \\
\text { G } \\
(n=99)\end{array}$ & $\begin{array}{c}\text { Unidade } \\
\text { H } \\
(n=93)\end{array}$ & $\begin{array}{c}\text { Total } \\
(n=579)\end{array}$ \\
\hline Idade (anos) & $26 \pm 7$ & $27 \pm 8$ & $25 \pm 8$ & $28 \pm 7$ & $27 \pm 7$ & $27 \pm 7$ & $27 \pm 7$ & $26 \pm 7$ & $27 \pm 7$ \\
\hline Adolescentes (\%) & 22 & 21 & 33 & 12 & 16 & 15 & 20 & 21 & 20 \\
\hline $\begin{array}{l}\text { Escolaridade }<8 \\
\text { anos }(\%)\end{array}$ & 20 & 42 & 6 & - & 28 & 34 & 44 & 38 & 32 \\
\hline $\begin{array}{l}\text { Pré-natal } \\
\text { presente (\%) }\end{array}$ & 85 & 93 & 89 & 92 & 92 & 98 & 91 & 91 & 91 \\
\hline $\begin{array}{l}\text { Gestação } \\
\text { múltipla (\%) }\end{array}$ & 27 & 21 & 14 & 18 & 14 & 7 & 16 & 15 & 16 \\
\hline $\begin{array}{l}\text { Síndrome } \\
\text { hipertensiva (\%) }\end{array}$ & 39 & 41 & 35 & 44 & 42 & 68 & 46 & 37 & 43 \\
\hline Diabetes (\%) & 5 & 3 & 6 & 2 & 2 & 12 & 5 & 9 & 5 \\
\hline $\begin{array}{l}\text { Infecção } \\
\text { periparto (\%) }\end{array}$ & 34 & 18 & 52 & 23 & 31 & 39 & 29 & 30 & 31 \\
\hline $\begin{array}{l}\text { Corticóide } \\
\text { pré-natal (\%) }\end{array}$ & 88 & 71 & 68 & 12 & 49 & 78 & 58 & 44 & 54 \\
\hline Parto cesárea (\%) & 63 & 66 & 61 & 68 & 72 & 85 & 69 & 60 & 68 \\
\hline
\end{tabular}

$\mathrm{AB}=$ atresia biliar.

com ou sem hemocultura positiva; uso de pelo menos uma dose de surfactante, independente da época e tipo; ventilação assistida (necessidade de pressão positiva contínua nas vias aéreas, ou CPAP, e/ou ventilação mecânica). O óbito foi classificado conforme a época de ocorrência.

Procedeu-se à análise descritiva de todas as variáveis de acordo com a unidade de nascimento. A seguir, compararam-se as características dos recém-nascidos que faleceram antes de 168 horas de vida em relação aos que sobreviveram após este período. Tal comparação foi feita, para as variáveis qualitativas, com o qui-quadrado ou teste exato de Fisher e, para as quantitativas, por meio do teste $t$ ou de Mann-Whitney. O passo seguinte foi comparar a freqüência de óbitos neonatais precoces entre as oito unidades, controlada pelo índice de gravidade neonatal (SNAPPE-II). Finalmente, para verificar os fatores associados ao óbito intrahospitalar precoce, efetuou-se a regressão logística com a estratégia passo a passo. Para a regressão logística, os fatores independentes analisados foram todos aqueles que mostraram $\mathrm{p}<0,20$ à análise univariada. Diante de possíveis covariadas, escolheram-se as variáveis com maior relevância clínica. Assim, optou-se por iniciar o modelo com a variável "idade gestacional", excluindo-se o "peso ao nascer" e, da mesma forma, preferiu-se analisar o "Apgar no $5^{\circ}$ minuto", excluindo-se as variáveis "ventilação com pressão positiva" e "reanimação avançada", assim como se optou por incluir a variável "síndrome do desconforto respiratório" em vez de "pelo menos uma dose de surfactante".

O cálculo amostral levou em conta a necessidade de 10 a 15 pacientes para cada uma das 20 variáveis independentes a serem analisadas no modelo de regressão logística. Assim, previu-se a inclusão de pelo menos 300 recém-nascidos no estudo.

Para toda a análise, o estatístico era cego em relação à identidade das unidades, o software empregado foi o SPSS 12.0 e considerou-se significante $p<0,05$.

\section{Resultados}

No período de 01/06/04 a 31/05/05, nasceram vivas 17.219 crianças nas oito unidades, das quais 579 (3\%) eram prematuros com idade gestacional entre $230 / 7$ e $336 / 7$, peso de 400 a 1.500 g, sem malformações congênitas maiores. As características maternas, de nascimento e a evolução clínica nas primeiras 72 horas dos pacientes nascidos nas oito unidades foram heterogêneas.

Quanto às características maternas (Tabela 1), as freqüências mínimas e máximas nas unidades de A-H foram: mães adolescentes (12-33\%), escolaridade inferior a oito anos (6-44\%), pré-natal presente (85-98\%), gestação múltipla (7-27\%), síndrome hipertensiva $(35-68 \%)$, diabetes (2-12\%), infecção periparto (18-52\%), uso de corticóide antenatal (12-88\%) e parto cesárea (60-85\%).

Em relação às características dos pacientes ao nascimento (Tabela 2), as freqüências mínimas e máximas nas unidades de $\mathrm{A}-\mathrm{H}$ foram: idade gestacional entre 23-27 semanas $(22-37 \%)$, peso $<1.000 \mathrm{~g}(32-55 \%)$, pequenos para a idade gestacional (24-55\%), sexo masculino (41-59\%), ventilação na sala de parto (61-77\%), reanimação avançada (0-26\%), 
Tabela 2 - Características dos pacientes ao nascer nas oito unidades estudadas

\begin{tabular}{|c|c|c|c|c|c|c|c|c|c|}
\hline & $\begin{array}{c}\text { Unidade } \\
\text { A } \\
(n=41)\end{array}$ & $\begin{array}{c}\text { Unidade } \\
\text { B } \\
(n=68)\end{array}$ & $\begin{array}{c}\text { Unidade } \\
\text { C } \\
(n=66)\end{array}$ & $\begin{array}{c}\text { Unidade } \\
\text { D } \\
(n=85)\end{array}$ & $\begin{array}{c}\text { Unidade } \\
\text { E } \\
(n=86)\end{array}$ & $\begin{array}{c}\text { Unidade } \\
\text { F } \\
(n=41)\end{array}$ & $\begin{array}{c}\text { Unidade } \\
\text { G } \\
(n=99)\end{array}$ & $\begin{array}{c}\text { Unidade } \\
\text { H } \\
(n=93)\end{array}$ & $\begin{array}{c}\text { Total } \\
(n=579)\end{array}$ \\
\hline IG (semanas) & $29,6 \pm 2,6$ & $28,4 \pm 2,5$ & $28,7 \pm 2,4$ & $29,2 \pm 2,9$ & $29,0 \pm 2,6$ & $28,6 \pm 2,6$ & $28,9 \pm 2,8$ & $28,6 \pm 3,2$ & $28,9 \pm 2,9$ \\
\hline IG $23-27$ semanas $(\%)$ & 22 & 37 & 35 & 25 & 28 & 32 & 32 & 33 & 31 \\
\hline PN (g) & $1.083 \pm 292$ & $1.017 \pm 217$ & $1.091 \pm 267$ & $994 \pm 281$ & $1.019 \pm 293$ & $989 \pm 294$ & $1.016 \pm 298$ & $965 \pm 311$ & $1.017 \pm 291$ \\
\hline PN 400-999 g (\%) & 32 & 47 & 38 & 47 & 47 & 44 & 50 & 55 & 46 \\
\hline Pequenos para a IG (\%) & 51 & 35 & 24 & 55 & 53 & 39 & 46 & 48 & 45 \\
\hline Sexo masculino (\%) & 44 & 57 & 55 & 47 & 43 & 41 & 46 & 59 & 50 \\
\hline VPP na sala de parto (\%) & 61 & 49 & 77 & 73 & 72 & 71 & 59 & 72 & 67 \\
\hline $\begin{array}{l}\text { Reanimação } \\
\text { avançada (\%) }\end{array}$ & 2 & 0 & 5 & 14 & 6 & 22 & 17 & 26 & 13 \\
\hline Apgar no $5^{\circ}$ minuto & $8 \pm 1$ & $8 \pm 1$ & $8 \pm 2$ & $5 \pm 3$ & $7 \pm 2$ & $8 \pm 2$ & $8 \pm 3$ & $8 \pm 3$ & $8 \pm 2$ \\
\hline Apgar $5^{\circ}$ minuto $<7(\%)$ & 5 & 10 & 15 & 26 & 26 & 7 & 20 & 19 & 18 \\
\hline $\begin{array}{l}\text { Surfactante na sala de } \\
\text { parto }(\%)\end{array}$ & 2 & 60 & 0 & 0 & 40 & 0 & 0 & 1 & 13 \\
\hline Óbito na sala de parto & 0 & 0 & 0 & $4(5 \%)$ & 0 & $1(2 \%)$ & $5(5 \%)$ & $9(10 \%)$ & $19(3 \%)$ \\
\hline
\end{tabular}

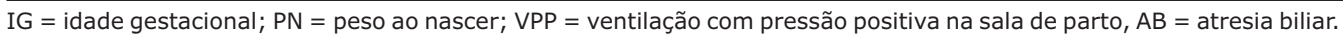

Apgar $<7$ no $5^{\circ}$ minuto (5-26\%) e surfactante na sala de parto (0-60\%).

Em termos da evolução clínica dos recém-nascidos nas primeiras 72 horas, nas unidades A-H (Tabela 3), as freqüências mínimas e máximas foram: maior gravidade clínica (SNAPPE-II $\geq 40$ ) (9-29\%), síndrome do desconforto respiratório (53-77\%), persistência do canal arterial (28-49\%), sepse (3-51\%), pelo menos uma dose de surfactante (32$68 \%$ ) e ventilação assistida (70-95\%).

Morreram 92 recém-nascidos antes de 168 horas de vida, dos quais 44 (48\%) antes de 24 horas e, destes, 19 faleceram na sala de parto. Dos recém-nascidos com 23 semanas $(n=19), 14$ faleceram até o sexto dia de vida. O mesmo ocorreu para 17 dos 25 nascidos com 24 semanas, para 16 dos 37

Tabela 3 - Evolução clínica dos recém-nascidos admitidos nas oito unidades estudadas (excluindo os 19 óbitos ocorridos na sala de parto)

\begin{tabular}{|c|c|c|c|c|c|c|c|c|c|}
\hline & $\begin{array}{c}\text { Unidade } \\
\text { A } \\
(n=41)\end{array}$ & $\begin{array}{c}\text { Unidade } \\
\text { B } \\
(n=68)\end{array}$ & $\begin{array}{c}\text { Unidade } \\
\qquad \begin{array}{c}C \\
(n=66)\end{array}\end{array}$ & $\begin{array}{c}\text { Unidade } \\
\text { D } \\
(n=81)\end{array}$ & $\begin{array}{c}\text { Unidade } \\
\text { E } \\
(n=86)\end{array}$ & $\begin{array}{c}\text { Unidade } \\
\text { F } \\
(n=40)\end{array}$ & $\begin{array}{c}\text { Unidade } \\
\text { G } \\
(n=94)\end{array}$ & $\begin{array}{c}\text { Unidade } \\
\text { H } \\
(n=84)\end{array}$ & $\begin{array}{c}\text { Total } \\
(n=560)\end{array}$ \\
\hline SNAPPE-II $\geq 40(\%)$ & 10 & 16 & 14 & 9 & 29 & 24 & 19 & 27 & 19 \\
\hline SDR (\%) & 56 & 66 & 77 & 42 & 49 & 68 & 53 & 60 & 58 \\
\hline PCA (\%) & 49 & 41 & 44 & 28 & 34 & 32 & 32 & 31 & 37 \\
\hline $\begin{array}{l}\text { Sepse antes } \\
\text { de } 72 \mathrm{~h}(\%)\end{array}$ & 44 & 3 & 37 & 51 & 49 & 35 & 18 & 19 & 31 \\
\hline $\begin{array}{l}\text { Surfactante } \geq 1 \\
\text { dose (\%) }\end{array}$ & 54 & 68 & 48 & 32 & 64 & 63 & 39 & 42 & 49 \\
\hline $\begin{array}{l}\text { Ventilação } \\
\text { assistida (\%) }\end{array}$ & 90 & 84 & 95 & 89 & 94 & 92 & 70 & 93 & 88 \\
\hline Óbito < $168 \mathrm{~h}$ & $\begin{array}{l}2 / 41 \\
(5 \%)\end{array}$ & $\begin{array}{l}5 / 68 \\
(7 \%)\end{array}$ & $\begin{array}{l}5 / 66 \\
(8 \%)\end{array}$ & $\begin{array}{l}11 / 85 \\
(13 \%)\end{array}$ & $\begin{array}{l}12 / 86 \\
(14 \%)\end{array}$ & $\begin{array}{c}6 / 41 \\
(15 \%)\end{array}$ & $\begin{array}{l}22 / 99 \\
(22 \%)\end{array}$ & $\begin{array}{l}29 / 93 \\
(31 \%)\end{array}$ & $\begin{array}{l}92 / 579 \\
(16 \%)\end{array}$ \\
\hline Óbito $<24 \mathrm{~h}$ & 0 & 0 & 0 & 9 & 6 & 3 & 13 & 13 & 44 \\
\hline
\end{tabular}

PCA = persistência do canal arterial; SDR = síndrome do desconforto respiratório; SNAPPE II = Score for Neonatal Acute Physiology, Perinatal Extension, Version II. 
Tabela 4 - Características dos prematuros que evoluíram para óbito antes de 168 horas versus os que sobreviveram após este período

\begin{tabular}{|c|c|c|c|}
\hline & \multicolumn{2}{|c|}{ Óbito hospitalar antes de 168 horas } & \multirow[b]{2}{*}{$\mathbf{p}$} \\
\hline & $\operatorname{Sim}(n=92)$ & Não (n = 487) & \\
\hline Idade materna (anos) & $26,7 \pm 7,0$ & $26,8 \pm 7,2$ & 0,919 \\
\hline Pré-natal presente & $81(88 \%)$ & $445(91 \%)$ & 0,394 \\
\hline Gestação múltipla & $23(25 \%)$ & $71(15 \%)$ & 0,013 \\
\hline Síndrome hipertensiva & $21(23 \%)$ & $226(46 \%)$ & $<0,001$ \\
\hline Diabetes & $5(5 \%)$ & $25(5 \%)$ & 0,801 \\
\hline Infecção periparto & $29(32 \%)$ & $149(31 \%)$ & 0,918 \\
\hline Corticóide antenatal & $34(37 \%)$ & $274(56 \%)$ & $<0,001$ \\
\hline Parto cesárea & $42(46 \%)$ & $349(72 \%)$ & $<0,001$ \\
\hline IG 23-27 semanas & $67(73 \%)$ & $146(30 \%)$ & $<0,001$ \\
\hline Peso ao nascer $<1.000 \mathrm{~g}$ & $77(84 \%)$ & $192(39 \%)$ & $<0,001$ \\
\hline Pequenos para a IG & $30(33 \%)$ & $231(47 \%)$ & 0,009 \\
\hline Sexo masculino & $49(53 \%)$ & $239(49 \%)$ & 0,462 \\
\hline VPP na sala de parto & $80(87 \%)$ & $308(63 \%)$ & $<0,001$ \\
\hline Reanimação avançada & $36(39 \%)$ & $37(8 \%)$ & $<0,001$ \\
\hline Apgar $5^{\circ}$ minuto $<7$ & $48(52 \%)$ & $56(11 \%)$ & $<0,001$ \\
\hline SNAPPE-II $\geq 40$ & $41 / 69(59 \%)$ & $64 / 481(13 \%)$ & $<0,001$ \\
\hline SDR & $64(70 \%)$ & $263(54 \%)$ & $<0,001$ \\
\hline PCA & $15(16 \%)$ & $190(39 \%)$ & 0,001 \\
\hline Sepse antes de $72 \mathrm{~h}$ & $29(32 \%)$ & $144(30 \%)$ & 0,131 \\
\hline Surfactante $\geq 1$ dose & $55(60 \%)$ & $231(47 \%)$ & 0,030 \\
\hline
\end{tabular}

IG = idade gestacional; PCA = persistência do canal arterial, SDR = síndrome do desconforto respiratório; SNAPPE-II = Score for Neonatal Acute Physiology, Perinatal Extension, Version II, descrito sobre o total de pacientes com o dado; VPP = ventilação com pressão positiva na sala de parto.

neonatos com 25 semanas, para nove dos 43 nascidos com 26 semanas e para 11 dos 54 pacientes com 27 semanas de idade gestacional. Entre 28 e 33 semanas, nasceram 401 crianças, das quais 25 (6\%) morreram na primeira semana de vida. O óbito neonatal precoce variou entre $5 \%$ na unidade $\mathrm{A}$ até $31 \%$ na unidade $\mathrm{H}$ (Tabela 3 ).

A comparação entre os 92 neonatos que faleceram antes de 168 horas e os sobreviventes mostrou maior freqüência, entre os óbitos, de gestação múltipla, idade gestacional de 23-27 semanas, peso ao nascer $<1.000 \mathrm{~g}$, necessidade de ventilação e de reanimação avançada na sala de parto, Apgar $<7$ no $5^{\circ}$ minuto, SNAPPE-II > 39, presença de síndrome do desconforto respiratório e uso de surfactante. Por outro lado, os recém-nascidos que faleceram antes de 168 horas apresentaram menor freqüência de síndrome hipertensiva materna, uso de corticóide antenatal, parto cesárea, pequeno para a idade gestacional e persistência do canal arterial (Tabela 4).

Ao analisar a freqüência de óbitos antes de 168 horas de acordo com a unidade de nascimento, controlou-se este desfecho pela gravidade clínica dos pacientes nas primeiras 12 horas de vida, ou seja, pelo SNAPPE-II. Observou-se que cada ponto a mais do escore aumentou em 7\% (IC95\% 5-8) a chance de óbito nos 550 (95\%) recém-nascidos para os quais essa informação estava disponível. Para analisar a mortalidade das oito unidades controlada pelo SNAPPE-II, escolheu-se como referência, dentre as unidades com menor mortalidade absoluta (unidades A, B e C, Tabela 3), aquela para a qual as comparações mostraram intervalos de confiança mais estreitos, ou seja, a unidade B, obtendo-se as seguintes odds ratio (OR) e intervalos de confiança: 1,1 (IC95\% 0,2-6,9) para A versus B; 1,2 (IC95\% 0,3-5,2) para Cversus B; 1,0 (IC95\% 0,2-4,2) para D versus B; 0,8 (IC95\% $0,2-3,2)$ para E versus $\mathrm{B} ; 1,4$ (IC95\% 0,3-6,4) para $\mathrm{F}$ versus B; 2,4 (IC95\% 0,7-8,3) para G versus B e 3,8 (IC95\% 1,1 a $12,8)$ para $\mathrm{H}$ versus $\mathrm{B}$.

A análise multivariada para o desfecho óbito neonatal intra-hospitalar precoce mostrou associação com os seguintes fatores independentes: idade gestacional de 23-27 semanas (OR $=5,0 ;$ IC95\% 2,7-9,4), ausência de síndrome hipertensiva (OR = 1,9; IC95\% 1,0-3,7), escore de Apgar entre 0-6 no $5^{\circ}$ minuto ( $O R=2,8$; IC95\% 1,4-5,4), presença de síndrome do desconforto respiratório (OR = 3,1; IC95\% 
1,4-6,6) e unidade. Os nascidos na unidade $\mathrm{H}$ mostraram 7,84 (IC95\% 1,57-39,17) vezes o risco de óbito precoce em relação à unidade $A$, controlando-se as outras variáveis do modelo. $O$ ajuste do modelo final mostrou-se adequado segundo o teste de Hosmer \& Lemeshow $(p=0,629)$.

\section{Discussão}

Este estudo multicêntrico mostra haver diferenças importantes entre as unidades avaliadas quanto à mortalidade neonatal precoce em recém-nascidos entre 23 e 33 semanas de idade gestacional, com peso entre 400 e $1.500 \mathrm{~g}$ e sem malformações congênitas maiores. Tais diferenças persistem ao se controlar os dados pela gravidade clínica dos pacientes e por outros fatores de risco perinatais associados ao óbito.

A população avaliada englobou recém-nascidos no extremo da viabilidade fetal. A decisão de incluir neonatos entre 23 e 25 semanas deveu-se à sua sobrevida, verificada nos estudos de redes epidemiológicas de países desenvolvi$\operatorname{dos}^{8,15,16}$, embora haja grande discussão a respeito da viabilidade, qualidade de vida futura e custos do cuidado a estes recém-nascidos ${ }^{17}$. De fato, dos 92 óbitos neonatais precoces analisados, 47 ocorreram em pacientes entre 23 e 25 semanas, delimitando a viabilidade nas maternidades estudadas ao redor de 26 semanas de idade gestacional. Ainda no que concerne à população de estudo, optou-se por excluir os prematuros tardios por se tratar de grupo com padrão de morbidade e mortalidade específico, bastante distinto dos nascidos até 33 semanas ${ }^{18,19}$. Os pacientes portadores de anomalias congênitas maiores também não foram incluídos, uma vez que a presença de malformações, por si só, leva a um aumento da chance de óbito ${ }^{2}$. Finalmente, estudaram-se apenas os nascidos nas próprias maternidades, não sendo analisados os pacientes externos, transportados para as unidades avaliadas, pois se sabe que a mortalidade dos últimos é significativamente mais elevada ${ }^{20}$.

Vale ressaltar que os resultados apresentados retratam hospitais universitários, de referência para a gestação de risco e que pertencem ao Sistema Único de Saúde. É interessante notar que o perfil dos óbitos neonatais brasileiros pode se modificar de acordo com a natureza jurídica do hospital. Em Belo Horizonte, investigação de base populacional entre 2000 e 2003 determinou a existência de três perfis distintos para o óbito neonatal: nos hospitais da rede privada, ocorreram óbitos dificilmente passíveis de prevenção (malformações e extremo baixo peso); nos hospitais contratados/conveniados ao Sistema Único de Saúde, os óbitos eram, em sua maioria, preveníveis (recém-nascidos com peso $>2.500 \mathrm{~g}$, que faleceram por processos hipóxicos e infecções); enquanto nos hospitais próprios do Sistema Único de Saúde, o perfil de mortes foi misto ${ }^{21}$.

Antes de analisar a mortalidade neonatal precoce como um todo, é necessário verificar as características das mães e seus recém-nascidos atendidos nas diferentes unidades, uma vez que várias publicações de redes epidemiológicas evidenciam que diferenças significantes nas características neonatais, de morbidades e de práticas assistenciais podem influenciar os desfechos estudados ${ }^{22-24}$. O presente estudo comprova estas diferenças. Em termos de práticas assistenciais, o uso de corticóide antenatal variou de $12 \%$ na unidade D (embora $92 \%$ das gestantes tivessem acompanhamento pré-natal) até $88 \%$ na unidade $A$. Ao nascimento, os procedimentos de reanimação avançada não foram realizados na unidade $B$ e se fizeram necessários em $26 \%$ dos pacientes da unidade $\mathrm{H}$, embora a idade gestacional e o peso ao nascer fossem semelhantes em ambas as unidades. Observa-se ainda que apenas as unidades $B$ e $E$ administraram surfactante nos primeiros minutos de vida, na sala de parto, e que o uso de pelo menos uma dose de surfactante variou de $32 \%$ na unidade $D$ até $68 \%$ na unidade $B$. Finalmente, no que se refere à ventilação assistida, a variação também foi notada, embora de menor monta comparada às outras práticas avaliadas. Assim, não é de se estranhar que o percentual de óbitos variasse entre as oito unidades, mesmo quando controlado pelo índice de gravidade clínica, o SNAPPE-II. Para diminuir a taxa de mortalidade neonatal precoce e a heterogeneidade das intervenções terapêuticas entre as diferentes unidades, o caminho a seguir parece ser o da implementação de programas de melhoria de qualidade dos processos de atendimento neonatal, baseados em quatro pontos-chave: estar receptivo para mudanças, habituar-se a aplicar práticas baseadas em evidências, avaliar de maneira sistemática e uniforme os resultados obtidos e aprender a trabalhar de modo colaborativo dentro da própria unidade e entre as diferentes unidades $^{25}$.

Dentre os fatores de risco independentemente associados ao óbito neonatal precoce, destaca-se, além da maternidade na qual ocorreu o nascimento, a baixa idade gestacional, a ausência de síndrome hipertensiva materna e a presença de asfixia perinatal. A maturidade fetal tem sido sempre a variável preditiva dominante em qualquer modelo de fatores associados ao óbito neonatal ${ }^{26}$. Vários trabalhos internacionais comprovam que, quanto mais baixa a idade gestacional, maior o risco de óbito. No Reino Unido, na Bélgica e na França, estudos realizados na década de 1990 com 1.976 nascidos com idade gestacional $\leq 26$ semanas mostraram que a mortalidade neonatal intra-hospitalar foi $91 \%$ com 23 semanas, $73 \%$ com 24 semanas, 53\% com 25 semanas e $46 \%$ com 26 semanas. Antes de 24 semanas, $68 \%$ morreram na sala de parto ${ }^{27}$. No Brasil, em 2004, 56\% dos 10.846 nascidos entre 22 e 27 semanas morreram até 6 dias de vida ${ }^{6}$, enquanto, no presente estudo, dos 178 nascidos entre 23 e 27 semanas, $38 \%$ faleceram antes de 168 horas. Desta forma, embora a mortalidade neonatal precoce dos prematuros entre 23 e 27 semanas encontrada seja inferior à nacional, nascer nesta faixa de idade gestacional aumentou em cinco vezes a chance de óbito neonatal precoce. Vale ressaltar que, para os nascidos entre 28 e 33 semanas, a freqüência de óbitos neonatais precoces neste estudo foi de apenas $6 \%$, inferior aos $20 \%$ 
encontrados para os nascidos entre 28 e 31 semanas no Brasil $^{6}$.

Um fator protetor para o óbito neonatal precoce foi a presença de síndrome hipertensiva durante a gestação. Outros estudos apontam para o mesmo achado. Evans et al. ${ }^{26}$ analisaram 11.498 nascidos de muito baixo peso entre 25 e 32 semanas na Austrália e Nova Zelândia, de 1998 a 2001. A hipertensão materna foi um fator protetor independente em relação ao óbito intra-hospitalar, diminuindo-o à cerca de metade $(O R=0,46$; IC95\% 0,36-0,50). Outra investigação multicêntrica, em recém-nascidos pequenos para a idade gestacional entre 24 e 42 semanas, admitidos em 17 unidades de terapia intensiva canadenses, mostrou que a hipertensão materna está associada à maior sobrevida. Uma possível explicação para estes achados relaciona-se às diferenças na nutrição placentária e transferência de oxigênio entre mães normotensas e hipertensas. Nestas, pode ocorrer uma redução na auto-regulação do fluxo arterial no espaço interviloso, com conseqüente aumento da perfusão e da passagem materno-fetal de nutrientes e oxigênio, levando ao aumento da sobrevida neonatal. Provavelmente, há um ponto em que esta perda de auto-regulação do fluxo interviloso se torna lesiva, aumentando a mortalidade materna e neonatal ${ }^{28}$. Neste contexto, ressalta-se a maior freqüência de pequenos para a idade gestacional entre os recém-nascidos que sobreviveram por, no mínimo, 1 semana.

O fato de a síndrome do desconforto respiratório manter-se como fator independente de risco para o óbito na primeira semana de vida indica falhas em vários níveis do cuidado perinatal. No período pré-parto, a baixa freqüência de uso de corticosteróide antenatal associa-se à maior incidência de síndrome do desconforto respiratório e mortalidade neonatal. Apenas 54\% dos pacientes abaixo de 34 semanas estudados receberam a medicação, enquanto, nos EUA, há mais de 10 anos, $79 \%$ das gestantes recebiam corticosterói$\mathrm{de}^{9}$. No período neonatal imediato, por sua vez, nota-se freqüência relativamente baixa do uso de surfactante, que pode evitar ou atenuar a gravidade da síndrome do desconforto respiratório: $49 \%$ dos prematuros aqui analisados fizeram uso de pelo menos uma dose de surfactante. Na Rede VermontOxford, dentre 26.007 pacientes nascidos em 1999 com peso entre 501 e $1.500 \mathrm{~g}, 62 \%$ receberam surfactante ${ }^{8}$. Finalmente, o fato de a síndrome do desconforto respiratório influir na mortalidade neonatal indica que há necessidade de melhorar o cuidado ao recém-nascido criticamente doente nas unidades analisadas.

Quanto à asfixia perinatal, observou-se que o escore de Apgar entre 0 e 6 no $5^{\circ}$ minuto se associou fortemente ao óbito neonatal precoce. Este achado é confirmado por outros estudos, destacando-se o de Casey et al., que avaliaram 13.399 produtos de gestação única entre 26 e 36 semanas, nascidos de 1988 a 1998 em um único centro estadunidense. O risco de morte neonatal foi 59 vezes maior quando o Apgar no 50 minuto foi de $0-3$ e 13 vezes maior para valores de 4-6, usando como referência escores de 7-10. O Apgar de $1^{\circ}$ minuto não se mostrou útil para predizer o risco de morte ${ }^{29}$.

Pode-se concluir que, embora as unidades universitárias estudadas sejam referência para o cuidado de pacientes criticamente doentes, a mortalidade neonatal precoce foi elevada para o padrão de países desenvolvidos, porém inferior à média nacional. Parte importante dos fatores de risco associados ao óbito neonatal precoce é passível de intervenção, como a melhora da vitalidade fetal ao nascer e a diminuição da incidência e/ou gravidade da síndrome do desconforto respiratório por meio do uso otimizado do corticóide antenatal e do surfactante pós-natal. Além disso, as diferenças de mortalidade encontradas entre as oito unidades apontam para a necessidade de identificar as possíveis melhores práticas e adotá-las de maneira mais uniforme em nosso meio.

\section{Agradecimentos}

À Adriana Sañudo, pela valiosa colaboração na análise estatística do estudo.

\section{Centros participantes e pesquisadores}

Hospital São Paulo, Universidade Federal de São Paulo Escola Paulista de Medicina (São Paulo, SP): Maria Fernanda Branco de Almeida, Ruth Guinsburg e Tatiana Furuyama.

Hospital de Clínicas de Ribeirão Preto, Universidade de São Paulo (Ribeirão Preto, SP): Francisco Eulógio Martinez, Maria Aparecida Rego e Marisa Márcia Mussi-Pinhata.

Hospital de Clínicas de Porto Alegre, Universidade Federal do Rio Grande do Sul (Porto Alegre, RS): Renato Soibelmann Procianoy e Rita de Cássia Silveira.

Hospital das Clínicas de São Paulo, Universidade de São Paulo (São Paulo, SP): Cléa Rodrigues Leone, Lílian Rodrigues dos Santos Sadeck, João Coriolano Rego Barros e Flávio Adolfo da Costa Vaz.

Centro de Atenção à Saúde da Mulher, Universidade Estadual de Campinas (Campinas, SP): Sérgio Tadeu Martins Marba e Gisele Marafon Lopes de Lima.

Hospital das Clínicas de Botucatu, Universidade Estadual Paulista (Botucatu, SP): Lígia Maria Sousa Suppo Rugolo, Cleide Enoir Petean Trindade e Adriana Sato.

Hospital São Lucas, Pontifícia Universidade Católica do Rio Grande do Sul (Porto Alegre, RS): Jorge Hecker Luz, Humberto Holmer Fiori, Manuel Rutkay Pereira e Renato Machado Fiori.

Instituto Fernandes Figueira, Fundação Osvaldo Cruz (Rio de Janeiro, RJ): José Maria de Andrade Lopes, Olga Bomfim, Maria Elisabeth Lopes Moreira e Saint Claire dos Santos Gomes Junior.

\section{Referências}

1. Joseph KS, Liston RM, Dodds L, Dahlgren L, Allen AC. Socioeconomic status and perinatal outcomes in a setting with universal access to essential health care services. CMAJ. 2007; 177:583-90. 
2. Mathews TJ, MacDorman MF. Infant mortality statistics from the 2004 period linked birth/infant death data set. Natl Vital Stat Rep. 2007; 55:1-32.

3. Kramer MS, Demissie K, Yang H, Platt RW, Sauve R, Liston R. The contribution of mild and moderate preterm birth to infant mortality. Fetal and Infant Health Study Group of the Canadian Perinatal Surveillance System. JAMA. 2000;284:843-9.

4. Ananth CV, Vintzileos AM. Epidemiology of preterm birth and its clinical subtypes. J Matern Fetal Neonatal Med. 2006;19:773-82.

5. Brasil. Ministério da Saúde. DATASUS. Informações de SaúdeEstatísticas Vitais- Mortalidade e Nascidos Vivos: nascidos vivos desde 1994. http://tabnet.datasus.gov.br/cgi/ deftohtm.exe?sinasc/cnv/nvuf.def. Acesso: 29.10.2007.

6. Brasil. Ministério da Saúde. DATASUS. Informações de SaúdeEstatísticas Vitais- Mortalidade e Nascidos Vivos: óbitos infantis - desde 1979. http://tabnet.datasus.gov.br/cgi/ deftohtm.exe?sim/cnv/infuf.def. Acesso: 29.10.2007.

7. Barros FC, Diaz-Rossello JL. The quality of care of very low birth weight babies in Brazil. J Pediatr (Rio J). 2007;83:5-6.

8. Horbar JD, Badger GJ, Carpenter JH, Fanaroff AA, Kilpatrick S, LaCorte $\mathrm{M}$, et al. Trends in mortality and morbidity for very low birth weight infants, 1991-1999. Pediatrics. 2002;110:143-51.

9. Fanaroff AA, Stoll BJ, Wright LL, Carlo WA, Ehrenkranz RA, Stark $A R$, et al. Trends in neonatal morbidity and mortality for very low birth weight infants. Am J Obstet Gynecol. 2007;196:147.e1-8.

10. Departamento de Neonatologia da Sociedade Brasileira de Pediatria. Infra-estrutura para atendimento integral ao recém-nascido. 2004. http://www.sbp.com.br/show_ item2.cfm?id_categoria=21\&id_detalhe=1636\&tipo_detalhe $=$ s. Access: 02.11.2007.

11. Ballard JL, Khoury JC, Wedig K, Wang L, Eilers-Walsman BL, Lipp R. New Ballard Score, expanded to include extremely premature infants. J Pediatr. 1991;119:417-23.

12. Alexander GR, Himes JH, Kaufman RB, Mor J, Kogan M. A United States national reference for fetal growth. Obstet Gynecol. 1996; 87:163-8.

13. Kattwinkel J. Textbook of Neonatal Resuscitation. 4th ed. Chicago, IL: American Academy of Pediatrics/American Heart Association; 2000.

14. Richardson DK, Corcoran JD, Escobar GJ, Lee SK. SNAP-II and SNAPPE-II: Simplified newborn illness severity and mortality risk scores. J Pediatr. 2001;138:92-100.

15. El-Metwally D, Vohr B, Tucker R. Survival and neonatal morbidity at the limits of viability in the mid 1990s: 22 to 25 weeks. J Pediatr. 2000;137:616-22.

16. Costeloe K, Hennessy E, Gibson AT, Marlow N, Wilkinson AR. The EPICure study: outcomes to discharge from hospital for infants born at the threshold of viability. Pediatrics. 2000; 106:659-71.
17. MacDonald $\mathrm{H}$; American Academy of Pediatrics. Committee on Fetus and Newborn. Perinatal care at the threshold of viability. Pediatrics. 2002;110:1024-7.

18. Jain L, Raju TN, editors. Late preterm pregnancy and the newborn. Clin Perinatol. 2006;33:751-972.

19. de Kleine $M J$, den Ouden $A L$, Kollee $L A$, Ilsen $A$, van Wassenaer AG, Brand $\mathrm{R}$, et al. Lower mortality but higher neonatal morbidity over a decade in very preterm infants. Paediatr Perinat Epidemiol. 2007;21:15-25.

20. Moro M, Figueras-Aloy J, Fernández C, Doménech E, Jiménez R, Pérez-Rodríguez J, et al. Mortality for newborns of birthweight less than 1,500 $\mathrm{g}$ in Spanish neonatal units (2002-2005). Am J Perinatol. 2007;24:593-601.

21. Drumond Ede F, Machado CJ, Franca E. Early neonatal mortality: An analysis of multiple causes of death by the Grade of Membership method. Cad Saude Publica. 2007;23:157-66

22. Richardson DK, Shah BL, Frantz ID 3rd, Bednarek F, Rubin LP, McCormick MC. Perinatal risk and severity of illness in newborns at 6 neonatal intensive care units. Am J Public Health. 1999; 89:511-6.

23. Horbar JD, Rogowski J, Plsek PE, Delmore P, Edwards WH, Hocker J, et al. Collaborative quality improvement for neonatal intensive care. NIC/Q Project Investigators of the Vermont Oxford Network. Pediatrics. 2001;107:14-22.

24. Vohr BR, Wright LL, Dusick AM, Perritt R, Poole WK, Tyson JE, et al. Center differences and outcomes of extremely low birth weight infants. Pediatrics. 2004;113:781-9.

25. Horbar JD, PIsek PE, Leahy K; NIC/Q 2000. NIC/Q 2000: establishing habits for improvement in neonatal intensive care units. Pediatrics. 2003;111:e397-410.

26. Evans N, Hutchinson J, Simpson JM, Donoghue D, Darlow B, Henderson-Smart D.Prenatal predictors of mortality in very preterm infants cared for in the Australian and New Zealand Neonatal Network. Arch Dis Child Fetal Neonatal Ed. 2007; 92:F34-40.

27. Walther FJ. Withholding treatment, withdrawing treatment, and palliative care in the neonatal intensive care unit. Early Hum Dev. 2005;81:965-72.

28. von Dadelszen $P$, Magee LA, Taylor EL, Muir JC, Stewart SD, Sherman $\mathrm{P}$, et al. Maternal hypertension and neonatal outcome among small for gestational age infants. Obstet Gynecol. 2005; 106:335-9.

29. Casey BM, McIntire DD, Leveno KJ. The continuing value of the Apgar score for the assessment of newborn infants. N Engl J Med. $2001 ; 344: 467-71$.

Correspondência:

Ruth Guinsburg

Rua Vicente Felix, 77/09

CEP 01410-020 - São Paulo, SP

Tel.: (11) 5579.4982

E-mail: ruthgbr@netpoint.com.br 